\title{
Experimental Study on Different Types of Curves for Ride Comfort in Automated Vehicles
}

\author{
Naohisa Hashimoto ${ }^{D},{ }^{1}$ Yusuke Takinami, ${ }^{1}$ and Makoto Yamamoto ${ }^{2}$ \\ ${ }^{1}$ Human Centered Mobility Research Center, National Institute of Advanced Industrial Science and Technology, Umezono, \\ Tsukuba 305-8568, Japan \\ ${ }^{2}$ Sanei Giken Cooperation, 3-26 Kaminoboricho, Naka-ku, Hiroshima-shi, Hiroshima-ken 730-0014, Japan \\ Correspondence should be addressed to Naohisa Hashimoto; naohisa-hashimoto@aist.go.jp
}

Received 24 May 2021; Revised 14 September 2021; Accepted 20 September 2021; Published 1 October 2021

Academic Editor: Peng Hang

Copyright (C) 2021 Naohisa Hashimoto et al. This is an open access article distributed under the Creative Commons Attribution License, which permits unrestricted use, distribution, and reproduction in any medium, provided the original work is properly cited.

\begin{abstract}
Vehicle automation is among the best possible solutions for traffic issues, including traffic accidents, traffic jams, and energy consumption. However, the user acceptance of automated vehicles is critical and is affected by riding comfort. In addition, human factors in automated vehicle control should be clear. This study evaluates the effect of different courses on driving comfort in automated vehicles using field experiments with 25 subjects. This study focused on lateral motion, but speed control was not targeted. Further, generating a path for obstacle avoidance and lane keeping, which have several constraining conditions, was also not targeted. Rendering a comfortable path is beneficial for developing an acceptable system as a car developer and for building new curves for automated or driving assistance systems from the perspective of construction. The automated vehicle drove at a speed of $30 \mathrm{~km} / \mathrm{h}$ on four courses, namely, clothoid, two types of spline curves, and arc, based on the real intersection. Each participant sat on both the driver and passenger seat and answered a questionnaire. The experimental data indicated the clothoid course to be the most comfortable, while the arc was most uncomfortable for a significance level of $1 \%$. These tendencies are applicable to driver and passenger seats, all genders, and experiences and will be beneficial for human factor research in automated vehicle control.
\end{abstract}

\section{Introduction}

An increase in urban traffic has led to increased traffic jams, traffic accidents, and air pollution, resulting in serious damage. Automated vehicles are expected to be a solution worldwide [1-3]. Automated vehicles can change the entire traffic system by making it more efficient and can contribute to the safety and reduction of $\mathrm{CO}_{2}$ emissions [4-6]. Furthermore, the introduction of automated vehicles could provide new services, such as autonomous riding shuttles, autonomous delivery, and autonomous valet parking. Many types of technologies for the introduction of completely automated vehicles have been developed, including position estimation, vehicle control, environmental recognition, public acceptance, human factors, infrastructure for automated vehicles, and laws [7-11].
The present study only considered lateral motion while speed control, generating a path for obstacle avoidance, and lane keeping, which have several constraining conditions, were not targeted. Safety is among the most important factors in the introduction of automated vehicles. Our automated vehicle employs the function of obstacle detection and avoidance, and when an obstacle is detected on the desired course, the priority of the control is to ensure safety to avoid the obstacle. In the experiments, there were no obstacles to the desired course. Thus, the obstacle avoidance function was inactive. In this study, we focused on comfort while ensuring safety. For the path for automated vehicle control, the clothoid curve has been extensively used according to several studies and the result of minimizing the maximum acceleration. Consequently, our hypothesis was that the clothoid curve was the most comfortable. However, 
research on automated driving for driving comfort is scarce. Further, the studies related to the human factor have mostly been evaluated using a simulator owing to the ease of setting up experimental conditions. However, evaluating our hypothesis in a simulation study was difficult, and thus, we decided to perform the experiments using a real vehicle to prove the hypothesis with persuasive results.

The present study evaluates the effect of several methods of constructing curves with the same turning radius on riding comfort. A real automated vehicle on test tracks was used, and riding comfort was evaluated by analyzing filled questionnaires. Although there exist several methods for constructing curves, and our experiments, four curves were constructed. The experimental vehicle, method of designing curves, experimental scenario, experimental results, and discussion have been explained in the following sections.

\section{Related Works}

Extensive research has been undertaken regarding driver human factors for automated vehicles.

Among the several human factors and the control of automated vehicles, comfort on control is critical for the introduction of automated vehicles [12].

Two types of controls for automated vehicles exist: longitudinal control [13-15] and lateral control. Du proposed a set of comfort-based velocity (longitudinal) control strategies based on driving safety. They insisted that the proposed strategy can potentially be applied to automated vehicles to improve the perceived quality of automated driving using field tests [13]. Reschka investigated the manner in which an autonomous vehicle can adapt its longitudinal control to changing road and weather conditions by calculating the proposed value and further provided an outlook on the way this parameter affected whole vehicle guidance [14]. Sohn proposed a longitudinal speed control strategy, which was called pulse and glide, and proved the improvement of driver satisfaction as well as fuel saving using the proposed method [15].

The present study focused on lateral control of riding comfort. The objective of lateral control is to allow the vehicle to travel on the desired course precisely. Researchers have proposed excellent lateral control algorithms [16-21], and using algorithms with optimal parameters, the vehicle can travel on the desired course with high accuracy. Park proposed a lateral control algorithm for automated vehicles using a proportional-integral-derivative (PID) controller [16] and validated the effectiveness of the proposed path at a curved path except at a low curvature path. Lee proposed a longitudinal control algorithm for a platoon of autonomous vehicles [17], which was evaluated using both simulators and real road experiments, proving its effectiveness. Chen proposed a path-following steering controller for an automated lane change system considering adaptive preview time. Further, using a simulator they validated that the proposed algorithm can effectively reduce the path-following error while reducing the lateral acceleration and jerk for a more comfortable lane change maneuver [18]. Yamakado focused on both longitudinal and lateral control of expert drivers while considering jerk information, and they confirmed that the model can emulate certain respect of the expert driver's control strategy for the trade-off between longitudinal and lateral acceleration [19]. Further, Alleyne performed a simulation and experimental study on automatic steering control and clarified that the steering actuator of the vehicle is a significant factor for model reference and PI-based control design [20]. Hingwe explained the design and experimental evaluation of a vehicle lateral controller for automated highway systems based on sliding mode control via experiments using a real passenger vehicle [21]. Except for lane-changing or obstacle avoidance, automated vehicles travel on the desired course. Hence, the driving comfort of traveling on the desired course depends on the course shape.

Several researchers have investigated human factors, particularly automated vehicles. Wang used a haptic interface for a driving assistance system for steering [22]. They concluded that the drivers tended to rely on haptic guidance to achieve better steering performance when visual feedback was limited via a simulation study [22]. Sentouh proposed a driver-automation cooperation-oriented approach, which is shared control between automated steering control and human driver on lane keeping. Their idea is based on a control design based on a human-in-the-loop vehicle system to reduce the conflict between the human driver and the system [23]. They proved the effectiveness of their proposed system experiments with real subjects.

Driving comfort has been investigated by several researchers. Chen investigated the human comfort of electrical vehicles and proposed a comfortable acceleration model [24]. Zuska experimented on the vibrational comfort of two passenger cars, wherein a defined and variable unbalance of a front wheel was set. They concluded that vibrational comfort was evaluated using the root mean square coefficient [25]. Butakov insisted on the importance of the personalization feature for automation and demonstrated the manner in which the methodology can be applied considering an example of adaptive cruise control and automatic lane-changing personalization [12]. Bae investigated the comfort experience lane-changing maneuver using the Bezier curve [26]. Xie proposed a personalized curve model for automated vehicles, insisting on the importance of the personalized curve for automated vehicles, and further evaluated the proposed model in a simulation study [27]. Lee researched the comfort zone for cyclists at different speeds and steering angles in field experiments [28]. To describe the importance of comfortable AV driving style, describing the importance of acceptance of $\mathrm{AV}$ is desirable, and it was found that those who did not prefer using AV preferred their own driving style. Oliveira reported user information on trust and acceptance of automated vehicles and insisted on the importance of improving trust and acceptance [29]. In addition, regarding the motion comfort of acceleration during driving, several studies insist that vibration is among the most important key factors for riding comfort. Mrad proposed a measurement technology to perform vibration analysis of a vehicle and a method that considered vibration with the suspension system. In addition, the best combination of rubber mounting, spring, and shock absorbers 
focusing on the user's well-being was proposed [30]. Sezgin investigated the effect of vehicle vibrations on passengers using the ISO 2631 standard [31], which sets the standard for mechanical vibration and shock-evaluation of human exposure to whole-body vibration. Further, several studies have been conducted on motion sickness [32]. Htike reported an effective control algorithm for motion sickness in automated vehicles [33], and Li evaluated motion sickness in automated vehicles using simulations and experiments [34]. However, motion sickness was not considered as the participants did not indicate any signs of it. Further, considering these results, in this study, the effect of vibration was not considered because the experimental course was completely flat and the difference among the four courses was not found in the experiment.

Regarding the method for creating the curvature using the features of a clothoid, it should be noted that the clothoid curve is familiar to the steering control. Sedighi proposed an algorithm for generating a parking path and confirmed the validity of the algorithm [35]. Lima proposed predictive control for autonomous vehicles using the features of a clothoid in a simulation study [36]. Berglund proposed a smooth path using the B-spline curve instead of an arc for automated mining vehicles, which can be effective in avoiding obstacles [37]. Further, De-rong used a spline curve to smoothly fit traffic road accidents. The feature of the spline curve, which is easy to calculate, was applied [38]. Wang studied optimizing trajectories in the intersection for automated vehicles, focusing on the trajectory choices for safety, energy savings, and comfort [39]. The present study focuses on human factors, wherein types of curvatures are most appropriate in automated vehicles for human drivers, and factors affecting the results were clarified in real-world experiments. Thus, based on the results of previous studies and results, it is proposed that driving comfort is among the most important issues in the introduction of automated vehicles.

\section{Experiments}

3.1. Experimental Vehicle. The experimental vehicle used in the experiments (Figure 1) is described in this section. This vehicle was modified from a conventional hybrid vehicle and the system configuration of the experimental vehicle is shown in Figure 1. It can be controlled via a PC through a control area network, and the system can obtain vehicle status information through an OBD-II port. Furthermore, two RTK-GPS sensors (Novatel OEM628), a gyro sensor (Tamagawaseiki TA7265), and five laser scanner sensors (ibeo LUX) for environmental recognition were installed.

3.2. Conditions of Experiments. The experiments were conducted on a test track at the National Institute of Advanced Industrial Science and Technology in Tsukuba, Japan. To prevent other undesirable effects, no other vehicles were present on the test track during the experiment. The experiments were conducted during the day, in sunny conditions to maintain consistent conditions.
3.3. Scenario of Experiments. Twenty-five subjects participated in the study. To eliminate age bias, five subjects from each age group (20s, 30s, 40s, 50s, and 60s) were chosen, and the average age of the drivers was 44.9. The experimental flow is illustrated in Figure 2.

First, the subjects received an explanation of the experiments and questionnaires regarding automated driving. The questionnaire regarding the characteristics of the subjects was constructed considering the following factors:

(1) Personal characteristics (age, gender, and history of driving and traffic accidents)

(2) Driving frequency, vehicle type for usual driving, and motion sickness

(3) Comments on automated driving systems

After completing the questionnaires, experiments with automated driving were conducted. In the experiments, one subject was asked to sit in the passenger seat, while another subject sat in the driver's seat or in the second-row passenger seat in the vehicle. All the subjects experienced both the driver and passenger seats. Half of the subjects sat in the driver's seat first and then the passenger's seat and vice versa. The velocity of the vehicle was maintained at approximately $8.3 \mathrm{~m} / \mathrm{s}(30.0 \mathrm{~km} / \mathrm{h})$. The effects of the varying speeds were clarified. In fact, preliminary experiments were performed with a small number of subjects before the experiments in this study by using the same experimental vehicle to evaluate the effect of speed. The preliminary experiments were performed under the same scenario at $20 \mathrm{~km} / \mathrm{h}$ and $40 \mathrm{~km} / \mathrm{h}$, and it was found that the tendency of the result was the same as the result at $30 \mathrm{~km} / \mathrm{h}$. Before the experiment, we explained the experimental process (including how to stop and steer the vehicle in an emergency) in detail to all subjects, sufficient time was devoted to preparing the experiments because we ensured a safe experimental environment for the automated vehicles. Consequently, the necessary time for the explanations to the subjects before conducting the experiments was long. In addition, because the budget was limited, employing the subjects was not possible. Moreover, owing to the course limitation, the experiments could not be performed at high speeds. Thus, we concentrated on a constant speed. Consequently, as employing more subjects was not possible, we determined the relationship results statistically; therefore, in this study, it is assumed that the number of participants was sufficient.

The control algorithms, including control parameters, are different among car companies and developers of automated vehicles, and they are classified. However, the original objective of the control algorithm was to travel the desired course without deviation, which was assumed as such. However, as we set the parameters of our control algorithm through preliminary experiments, the effect of the control algorithm was removed when evaluating the trajectory of the vehicle. The objective of this study is to evaluate the comfort of the trajectory, not the control algorithm; thus, it was considered that our experimental evaluation was complete for meeting the objective. However, the control algorithm should also be evaluated, particularly 

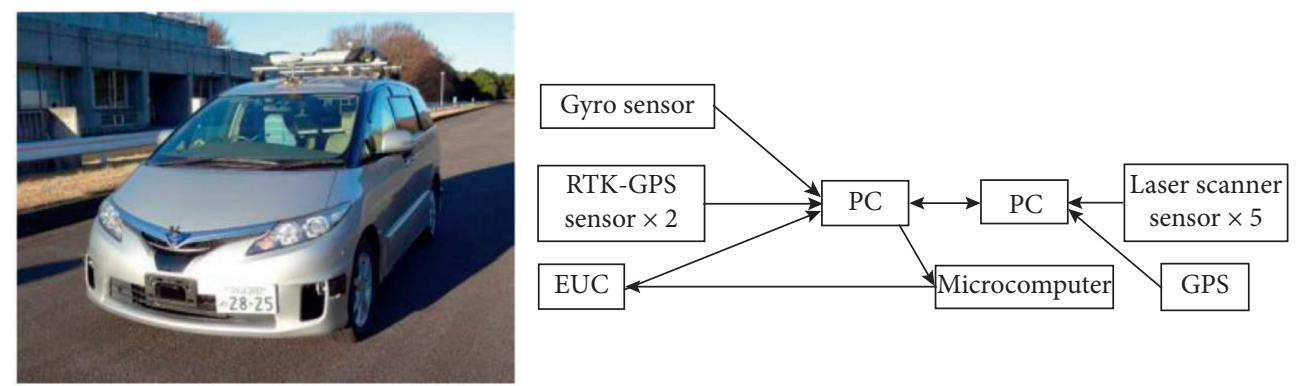

FIgURE 1: Picture and system configuration of the experimental vehicle.

\begin{tabular}{|l|}
\hline $\begin{array}{l}\text { 1. Explanation to the subject about the experiments } \\
\text { that are to be performed }\end{array}$ \\
\hline \hline $\begin{array}{l}\text { 2. Questionnaires about subject, automated vehicle, } \\
\text { driving style }\end{array}$ \\
\hline $\begin{array}{l}\text { 3. Experiments with automated driving in driver's seat } \\
\text { or passenger's seat }\end{array}$ \\
\hline 4. Questionnaires about the experiments \\
\hline $\begin{array}{l}\text { 5. Experiments with automated driving in passenger's } \\
\text { seat or driver's seat }\end{array}$ \\
\hline 6. Questionnaires about the experiments \\
\hline
\end{tabular}

FIgURE 2: Experimental flow.

for other situations, wherein the experimental vehicle cannot travel the desired course without deviation due to certain conditions (weather, vehicle, etc.). We plan to conduct an evaluation in this respect in a future study.

Each subject experienced four course patterns. In the experiments, the order of the four courses was set randomly and introduced as courses 1, 2, 3, and 4 to the subjects. Thus, the subjects could not recognize which path corresponded to each of the four path patterns. The course patterns were an arc, clothoid curve, B-spline curve 1, and B-spline curve 2 (explanations have been provided in the next section). The automated vehicle traveled along the target course autonomously, and four patterns in each set were obtained. The subjects experienced two sets in each seat; thus, they performed 16 rides in both seats. For safety, two staff members sat in the first-row passenger seat (next to the driver's seat) and the second-row passenger seat, and every subject completed a training course to prepare for possible emergencies. Furthermore, each subject was asked to control the experimental vehicle for possible emergencies, implying that each subject was responsible when sitting in the driver's seat. There were no accidental situations during the experiment. According to the SAE international definition, the level of the automated vehicle was set to two in our experiments when the subject sat in the driver's seat and four when the subject sat in the passenger's seat [40]. This automated vehicle experiment was performed at an SAE level of 2. Although we did not ask subjects to watch the road, they were responsible for their vehicles; thus, they watched the road.
After one set of experiments, the subjects completed questionnaires regarding the experiments. Thus, the subjects were asked to complete these questionnaires four times in both seats. The questionnaire for each set asked the following questions:

Q1: Which path is comfortable? (multiple selections possible)

Q2: Which path is the most comfortable?

Q3: Why did you choose that path?

Q4: Which path is uncomfortable? (multiple selections possible)

Q5: Which path is the most uncomfortable?

Q6: Why did you choose that path?

Q7: Any comments on the experiments?

3.4. Four Patterns of Target Courses. In this experiment, four target course patterns were used. Each course consisted of straight lines and two curves (Figure 3). The differences in the four course patterns were in the shape of the curves. The curves were an arc, clothoid, B-spline 1, and B-spline 2, hereafter referred to as R50, CL50, BSP1, and BSP2, respectively. All curves were based on a $50 \mathrm{~m}$ radius and the real crossing in Hiroshima, Japan (Figure 4). Regarding the clothoid curve, clothoid equations can be defined starting from the condition of the linear relation between the radius $(R)$ and length $(L)$ of the path (equation (1)) as 


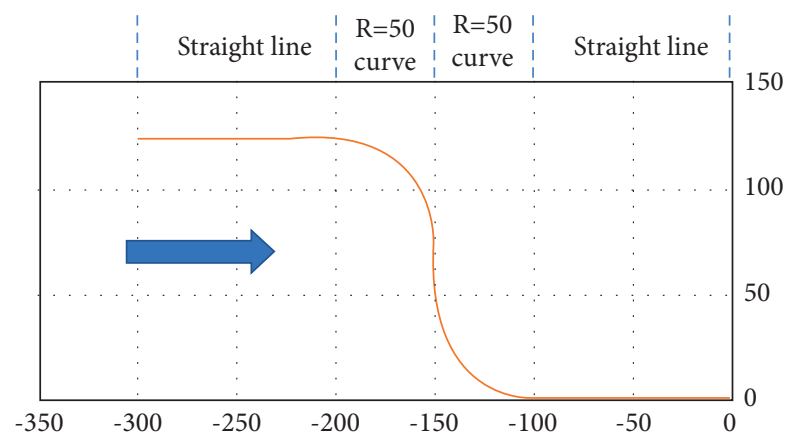

Figure 3: Experimental course of R50 (the arrow shows the direction of the vehicle).

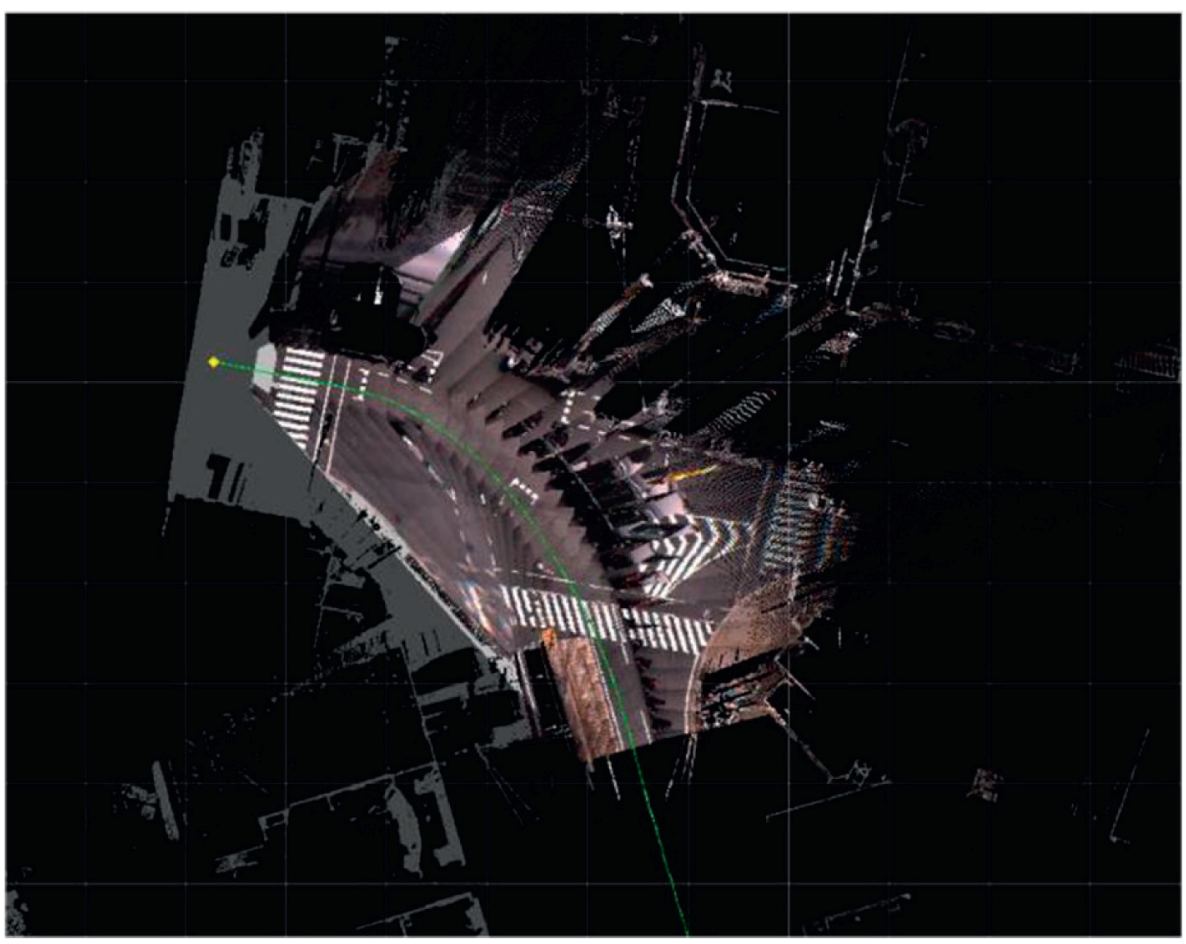

Figure 4: Sample used in Hiroshima, Japan.

$$
R * L=A^{2}
$$

where $R$ is the radius of curvature, $L$ is the length measured along the spiral curve from its initial position, and $A$ is the flatness or homothetic parameter of the clothoid.

In the present study, $R$ and $A$ were set to 50 and 35, respectively.

We employed a clothoid because the enables smooth steering control as indicated in several studies [41-44]. Consequently, we considered one hypothesis regarding comfort, which is that the clothoid curve is better for driving comfort. We conducted preliminary experiments with a few subjects and considering the results, we decided to prove our hypothesis with more subjects using real experimental vehicles. We believe that our experimental results validate our hypothesis.

BSP1 was prepared using the control points of the polyline (Figure 5(a)). Polylines were extracted along the channels (dots of the lane marker). Because spline conversion was performed to smoothen the polylines, the size of the BSP1 wobbles depends on the size of the original polyline rattle.

Further, BSP2 was made to pass three points: the initial point, midpoint, and terminal point of the clothoid curve (Figure 5(b)). Although BSP2 appears looser than the clothoid, on the initial and terminal points of the curve, there a sudden change from straight to curve and vice versa occurs. Typically, BSP2 requires higher transformation costs than BSP1 because the initial and terminal points of the clothoid must be recognized for generating BPS2.

Before performing the experiments with real subjects, preliminary experiments were performed for an objective evaluation. The experimental course for these experiments was set as shown in Figure 6. Each course consisted of straight lines and one curve. In the objective evaluation, evaluation of both left and right turns was not considered as 


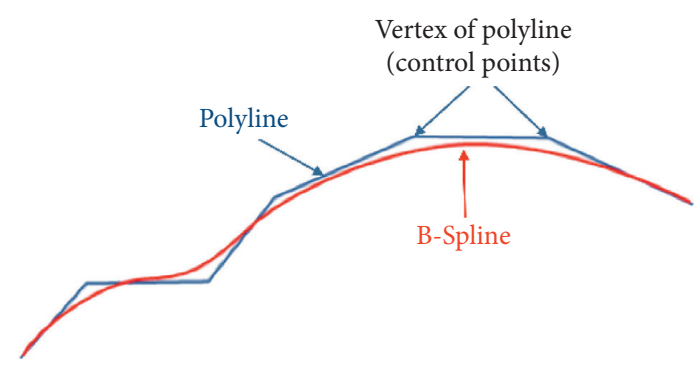

(a)

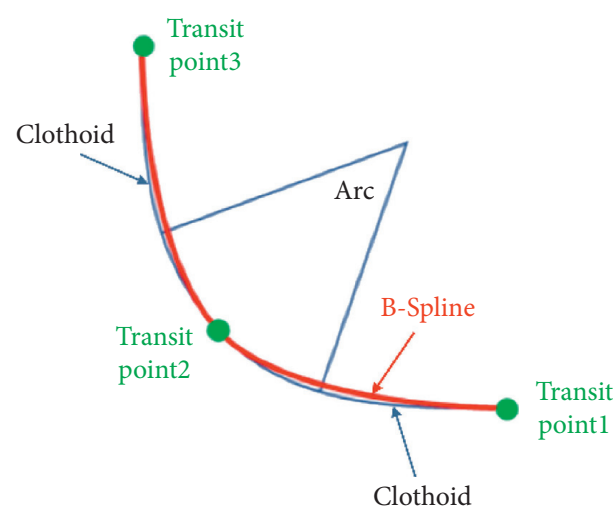

(b)

Figure 5: Method of forming B-spline curves. (a) B-spline 1 and (b) B-spline 2.

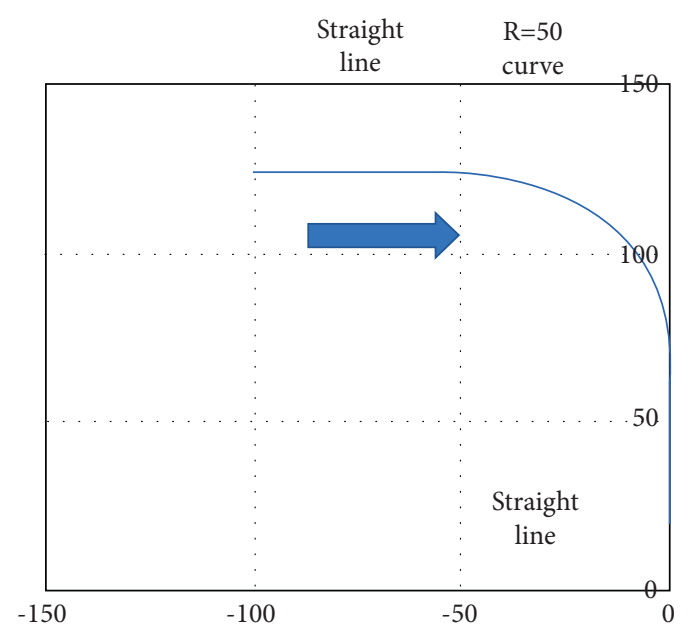

Figure 6: Experimental simple course for objective evaluations on the preliminary experiment.

necessary; thus, we employed a simple course. The lateral acceleration was evaluated, which was measured via an IMU 440 sensor, developed by Sumisei Sangyo. The scale factor accuracy of the sensor was $1 \%$. Figure 7 shows the result of the lateral acceleration of each course, and the result when traveling on the curvature. From the left, the results of clothoid, R50, BSP1, and BSP2 are shown. The maximum value of lateral acceleration with CL50 was the lowest among the four courses. In addition, the absolute peak of jerk was calculated to evaluate riding comfort, as shown in Table 1, wherein CL50 is shown to have the smallest value. However, the difference between them is very small. Several papers have proposed the relationship between the jerk value and riding comfort [45-47]. Considering this result, CL50 was assumed to be the most comfortable.

\section{Experimental Results and Discussion}

The results of Q1: "Which path is comfortable?" (multiple selections possible) of the questionnaire are shown in Figure $8(a)$. The maximum number of each value is 100 ; hence, as evident, approximately $70 \%$ of the subjects chose CL50, while approximately $60 \%$ chose BSP 2 . The results of

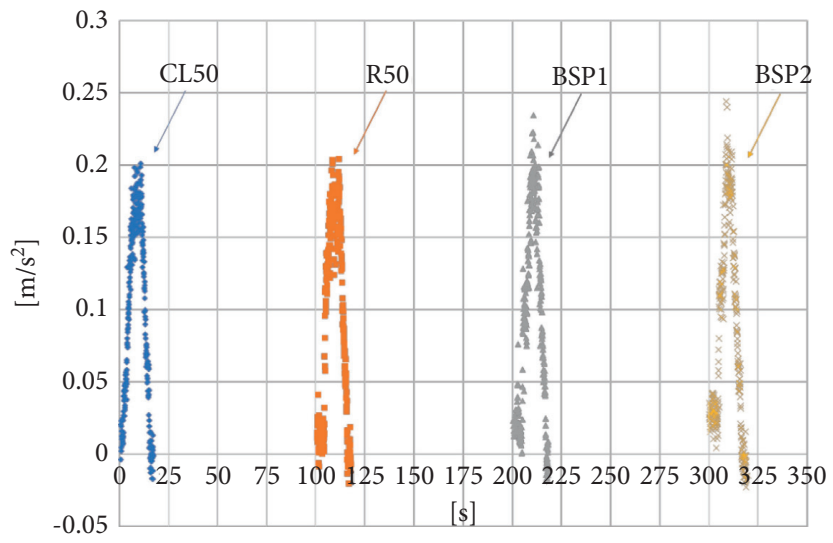

Figure 7: Experimental result for objective evaluation on the preliminary experiment (lateral acceleration).

TABle 1: Result of peak jerk on lateral acceleration on the preliminary experiment.

\begin{tabular}{lcccc}
\hline Course & CL50 & R50 & BSP1 & BSP2 \\
\hline $\begin{array}{l}\text { Peak absolute jerk on lateral } \\
\text { acceleration }\end{array}$ & 1.11 & 1.16 & 1.14 & 1.70 \\
\hline
\end{tabular}




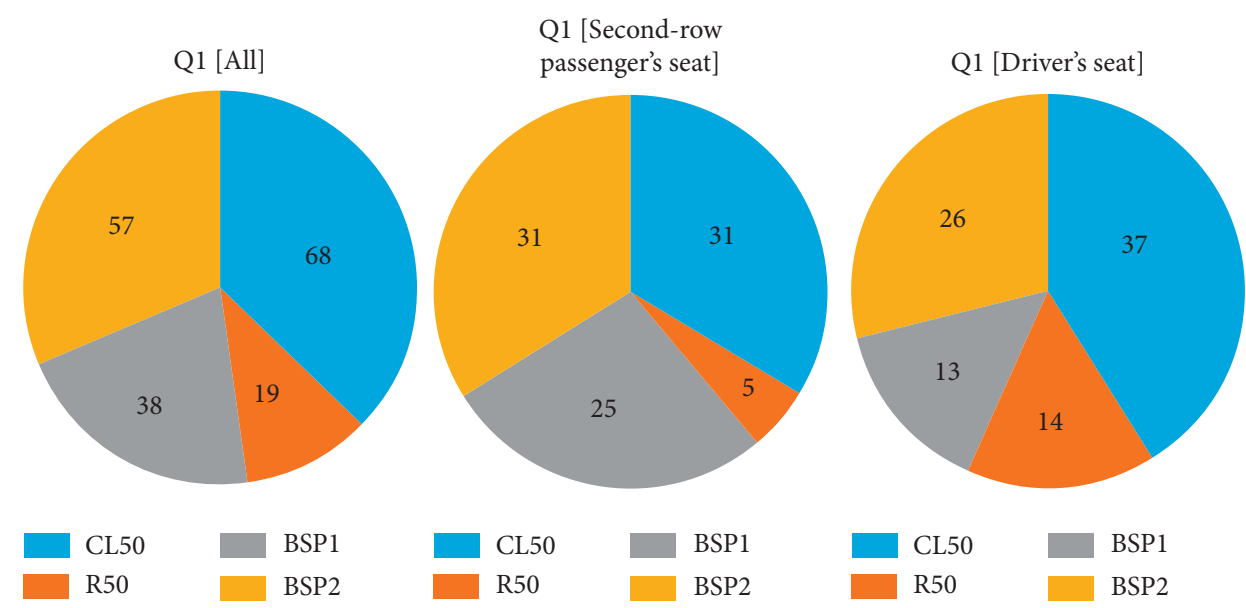

Figure 8: Questionnaire Result 1 (Q1: "Which path is comfortable? (multiple selections possible)"). CL50: clothoid, R50: arc, BSP1: B-spline 1, and BSP2: B-spline 2.

the difference in the subject's seat position are shown in Figure 8(c). Further, a comparison of the driver's seat and second-row passenger's seat results demonstrated that the latter tended not to choose R50.

Questionnaire results of Q2: "Which path is the most comfortable?" are shown in Figures 9(a)-9(c). It indicates that more than $50 \%$ of the subjects chose CL50 as the most comfortable path and that the clothoid curve was appropriate for a comfortable path. The questionnaire results for the riding positions are also shown in Figures 9. The CL50 was the most answered choice in both the driver and passenger seat positions, and subjects in the driver's seat tended to choose CL50 more than in the passenger seat. Regarding the first and second trials, the questionnaire results from the experience of tests are also shown in Figures 9(d) and 9(e). As explained in the experimental section, two tests were performed in each scenario. The " 1 st" and " 2 nd" are the orders of experiments. This result has the same tendency as the other results. Further, regarding gender, the questionnaire results by gender are also shown in Figures 9(f) and $9(\mathrm{~g})$. The difference between this result and the others is that the score of BSP1 is larger than that of BSP2, but the largest score is for CL50, the same as the others. In addition, the questionnaire results for nonelderly and elderly individuals are also shown in Figures 9(h) and 9(i). Elderly subjects chose different answers because they had different feelings. To evaluate the results statistically, we employed the chisquared test. The chi-squared test is commonly used in academic papers [48-50]. The formulation of the chisquared test is as follows:

$$
X_{c}^{2}=\sum \frac{\left(O_{i}-E_{i}\right)^{2}}{E_{i}},
$$

where $O_{i}$ is the observed numbers and $E_{i}$ is the expected numbers.

The results of the chi-squared test for Q2 are presented in Table 2. All questionnaire results for Q2 were satisfied with a $1 \%$ significance level. Further, subjects can identify four types of courses and decide which is the most comfortable. The questionnaire result of Q3: "Why did you choose that path?" is shown in Figure 10. The subjects provided reasons for choosing the path. Most subjects chose smooth control or less shaking. As clothoid is familiar with steering control [26] resulting in a small jerk, it is presumed that the subjects felt comfortable.

The questionnaire results of Q4: "Which path is uncomfortable? (multiple selections possible)" are shown in Figure 11. The maximum number of each value is 100 ; therefore, approximately $70 \%$ of the subjects chose R50, while approximately $60 \%$ chose BSP1. Regarding the driving position, subjects in the driver's seat chose BSP1 more than R50; although, the difference was small. It is presumed that subjects in the driver's seat can observe the shaking of the steering angle directly because the steering angle is subjected to minute shaking when traveling on BSP by the feature of BSP1.

The questionnaire results of Q5: "Which path is the most uncomfortable?" are shown in Figure 12. These results show that more than $50 \%$ of the subjects chose R50 as the most uncomfortable path. The results of the chi-squared test for Q5 are shown in Table 3. From these results, all questionnaire results of $\mathrm{Q} 5$, except for the driver seat, were satisfied with a $1 \%$ significance level.

Thus, the result indicates that R50 should not be applied as a comfortable path. Furthermore, subjects can identify the four types of courses and decide which is the most uncomfortable. The questionnaire results based on riding positions are also shown in Figures 12(a)-12(c). The choice of R50 is the maximum in both the driver and passenger seat positions, but in the former, the result of the chi-squared test is not satisfied with a $5 \%$ significance level. It is presumed that the subjects in the driver's seat could observe the steering angle directly (Figure 11). Further, the maximum number of R50 choices is the same as that of the others. Regarding the first and second trials, the questionnaire results from the experience of tests are shown in Figures 12(d) and 12(e). No differences were observed in the order of these results, which have the same tendency as the other results. The questionnaire's results by gender are also shown in Figures 12(f) and 12(g). Furthermore, these results show no differences between genders and have the same 


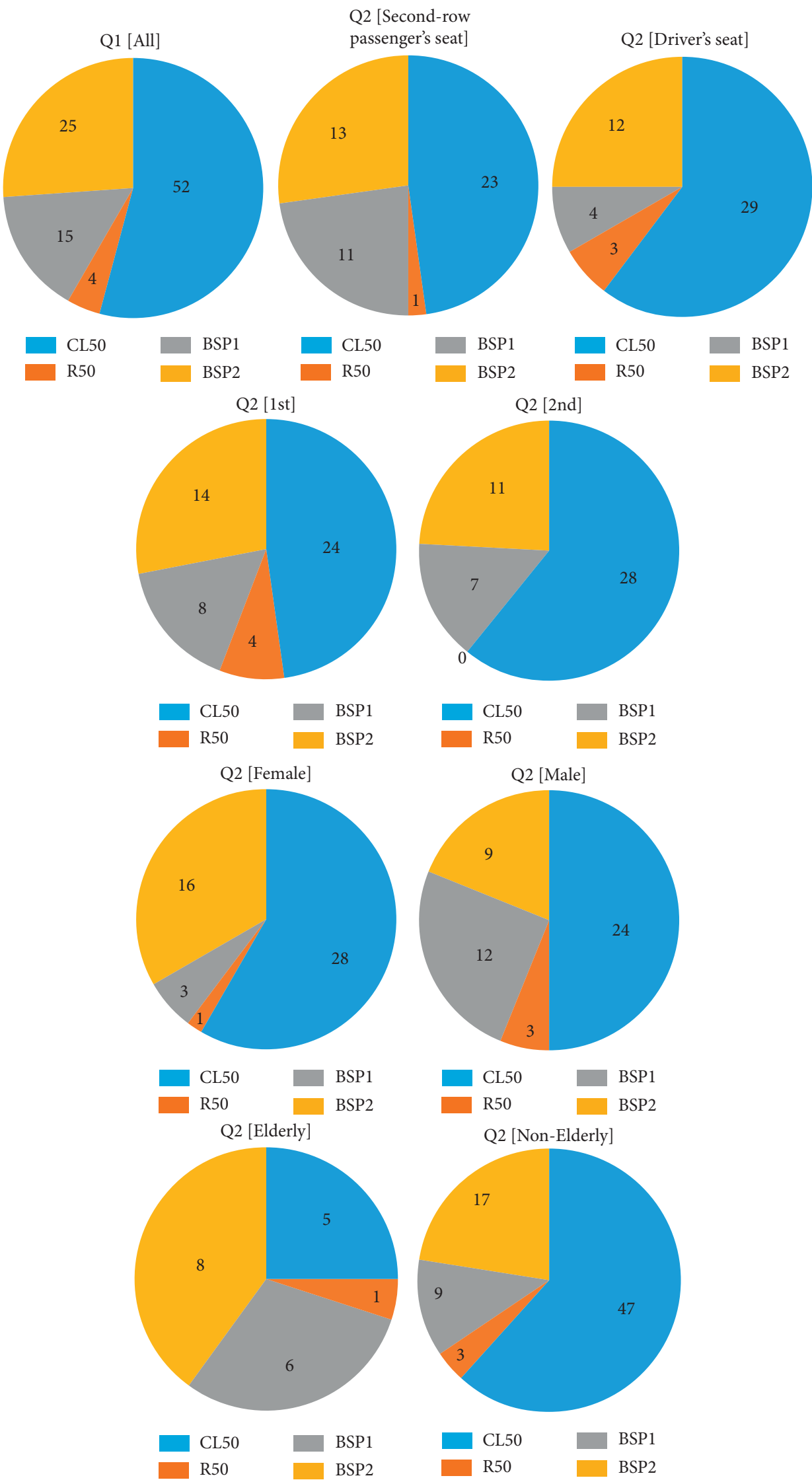

FIGURE 9: Questionnaire Result 2 (Q2: “Which path is the most comfortable?”). CL50: clothoid, R50: arc, BSP1: B-spline 1, and BSP2: B-spline 2. 
TABLE 2: Chi-squared test result of Q2: "Which path is the most comfortable?".

\begin{tabular}{lccccccccc}
\hline & \multicolumn{4}{c}{ (Which is the most comfortable?) degree of freedom $=3$} \\
& All & Ds & Rs & 1st & 2nd & Female & Male & Nonelderly & Elderly \\
\hline$X^{2}(7.82 \leq, p<0.05,11.34 \leq, p<0.01)$ & 52.75 & 24.08 & 20.33 & 18.16 & 36.96 & 39.5 & 19.5 & 60.2 & 5.2 \\
\hline
\end{tabular}

Ds: driver's seat; Rs: second-row passenger's seat.

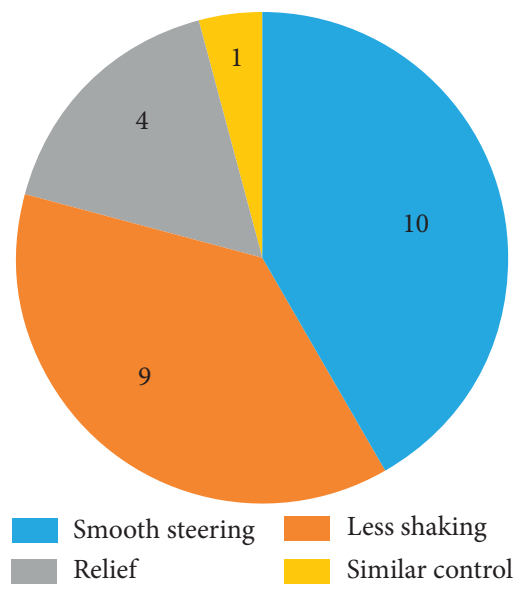

FIgURE 10: Questionnaire Result 3 (Q3: “Why did you choose that path?”).
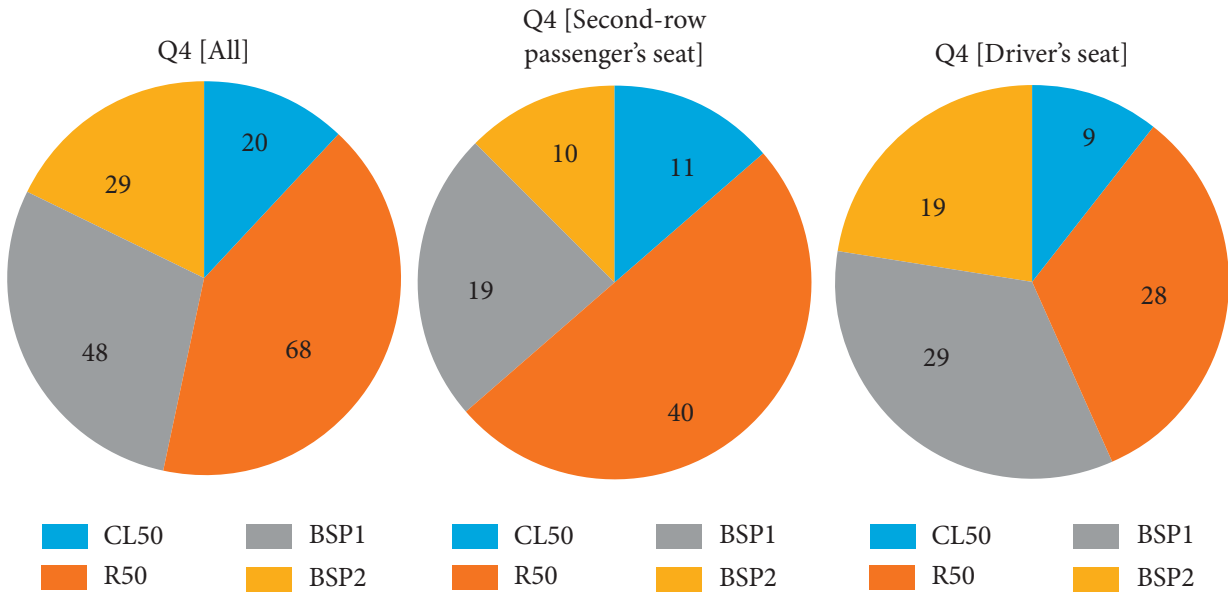

FIGURE 11: Questionnaire Result 4 (Q4: "Which path is the most uncomfortable (multiple selections possible)"). CL50: clothoid, R50: arc, BSP1: B-spline 1, and BSP2: B-spline 2.

tendency as the other results. Regarding age, the questionnaire results for the nonelderly and elderly are also shown in Figures 12(h) and 12(i). These results exhibit the same tendency as in Figure 11, showing a less statistical difference between the answers. However, the introduction of an automated driving system for elderly drivers should be considered from other perspectives. The questionnaire result of Q6: "Why did you choose that path?" is shown in Figure 13. The subjects also provided reasons for choosing the path. The reasons were primarily not smooth and shaking, which are not as complicated to comprehend as Q3. From this result and the chi-squared test results, it is presumed that it is easier to decide on a comfortable path than on an uncomfortable path.
The comments regarding the experiments are presented in Figure 14. The maximum number of comments was related to the number of repetitions. As shown in the results of the chi-squared test in Table 2, the value of the 2 nd was larger than that of the 1st. After one trial, the subjects experienced the automated vehicle and understood the difference better. Further, from the results regarding the riding position in Table 3, Figure 11, and Figure 14, it is presumed that observing the movement of the turning steering wheel could affect comfort; therefore, a human-machine interface for passengers should be installed to improve acceptability.

In addition, with respect to the feeling of safety, the results of this experiment included the feeling of safety, because if subjects felt unsafe on the course, they opted not 

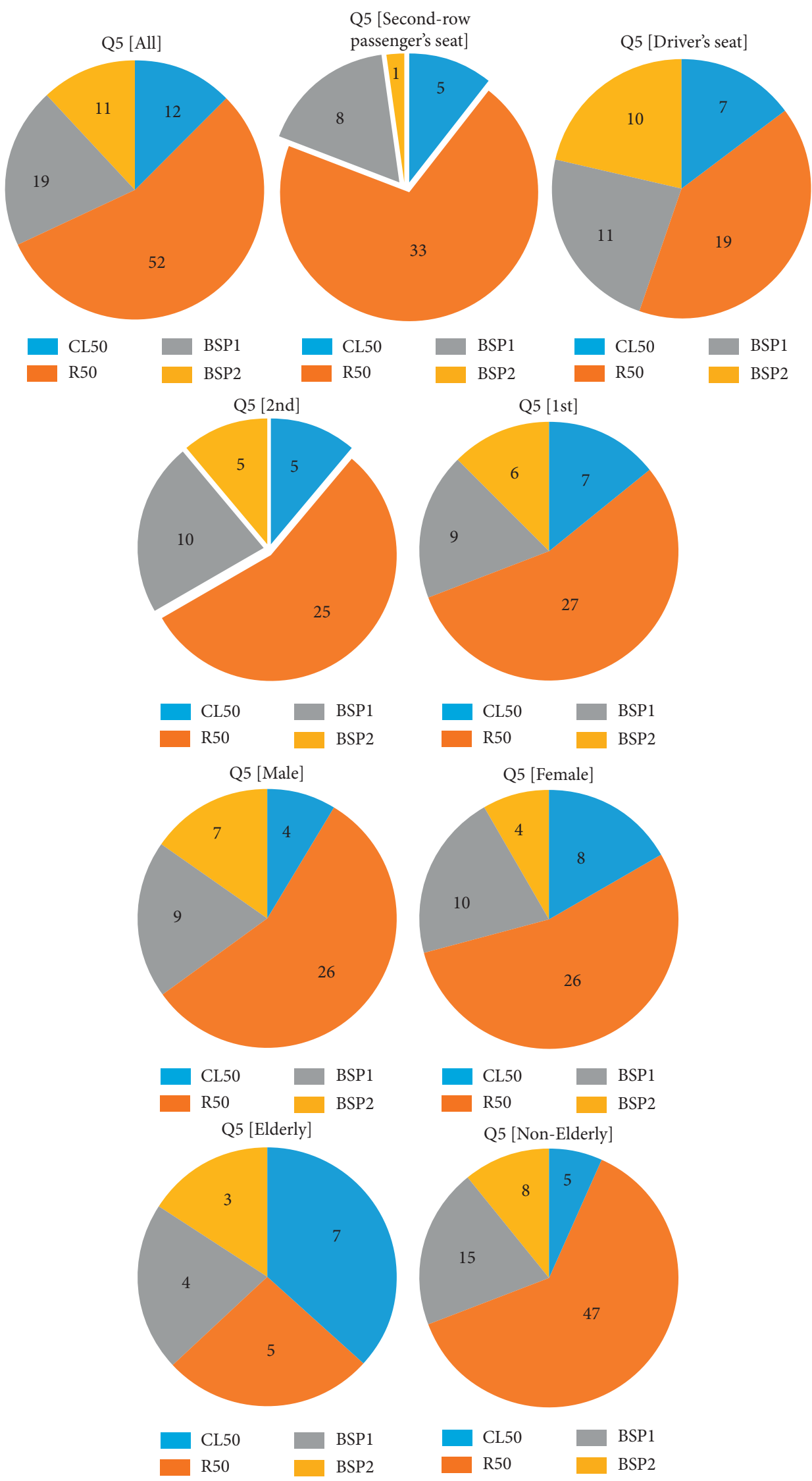

FIGURE 12: Questionnaire Result 5 (Q5: “Which path is the most uncomfortable?”). CL50: clothoid, R50: arc, BSP1: B-spline 1, and BSP2: B-spline 2. 
TABLE 3: Chi-squared test result of Q5: "Which path is the most uncomfortable?".

\begin{tabular}{lccccccccc}
\hline & \multicolumn{4}{c}{ (Which is the most uncomfortable?) degree of freedom $=3$} \\
& All & Ds & Rs & 1st & 2nd & Female & Male & Nonelderly & Elderly \\
\hline$X^{2}(7.82 \leq, p<0.05,11.34 \leq, p<0.01)$ & 47.70 & 6.70 & 53.34 & 24.06 & 23.89 & 23.33 & 18.16 & 59.56 & 1.84 \\
\hline
\end{tabular}

Ds: driver's seat; Rs: second-row passenger's seat.

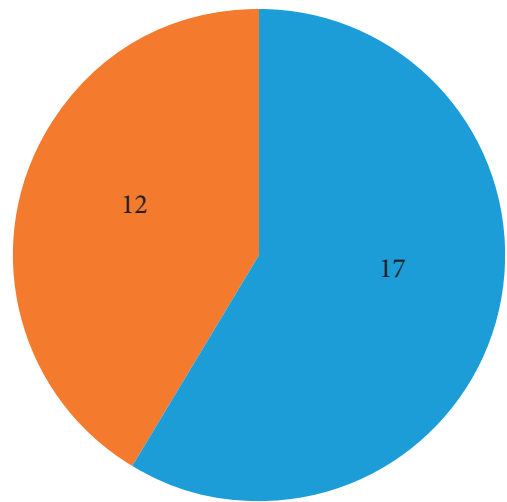

Unsmooth steering

Shaking

FIgURE 13: Questionnaire Result 6 (Q6: “Why did you choose that path?”).

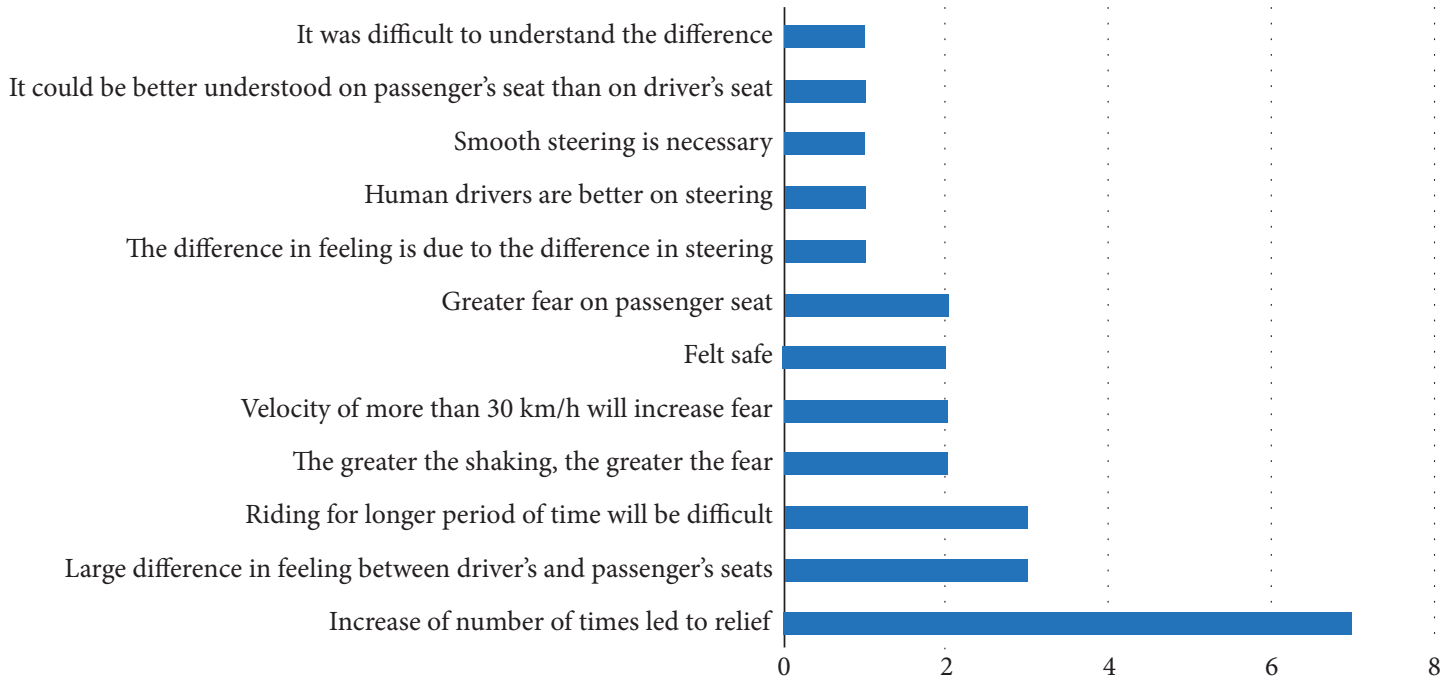

FIGURE 14: Questionnaire Result 7 (Q7: “Any comments on the experiments?”).

to choose the course. Thus, it is presumed that the results show that clothoid provides the best feeling of safety among the four courses. Hence, in future studies, the feeling of safety should be considered along with the function of obstacle avoidance. We also enquired about motion sickness in the questionnaire because the human factor of motion sickness might affect the results in our hypothesis. However, we could not find any relationship between the results and the factor of motion sickness as no subject reported the occurrence of motion sickness.

As explained in the scenario of experiments section, the level of automation of the experiments was set to two [40]. In the future, levels 3,4 , or 5 automated vehicles may be introduced [40]. A driver who rides on level 3, 4, or 5 automated vehicles can perform eyes-off road driving and nondriving tasks because the driver is not responsible for driving. In contrast, the driver who drives the level 2 automated vehicle is responsible for the vehicle. In Japan, only two types of vehicles with level 3 functions are produced. Level 4 and 5 automated vehicles have not yet been allowed by the government. Given the current situation in Japan, as level 3 automated vehicles are not yet well known, it is difficult for subjects to imagine level 3 automated vehicles. In Japan, several experiments related to level 2 automated vehicles and level 2 assistance systems are well known. In our experiments, we explained to the subjects that the level of 
automated vehicles was level 2 , and the responsibility was with the driver. In addition, for developing level 3 vehicles, the process of providing all explanations before using the vehicles is necessary, which results in requiring more time to prepare the experiments in real vehicles rather than in the simulator. Thus, in this study, it was difficult to perform the experiments with level 3,4 , or 5 automated vehicles. However, if the experimental vehicle is a level 3, 4, or 5 automated vehicle, the experimental result may be different, and performing experiments related to the eyes-off situation and nondriving task would be possible.

\section{Conclusions}

The present study investigated several courses to determine the most comfortable course for further introduction of automated vehicles. The conditions for the different curves based on real situations were assumed. This study evaluated the effect of different courses on driver comfort in automated vehicles through field experiments with 25 subjects. The automated vehicle drove at $30 \mathrm{~km} / \mathrm{h}$ on four different courses (clothoid, two types of spline curves, and arc). The experimental data indicated that the most comfortable course was clothoid, and the most uncomfortable course was an arc when the significance level was set at $1 \%$. The reasons for this were primarily smoothness while driving. Thus, the experimental results show that, for driving comfort, paths for automated vehicles should be clothoid and not arc. These tendencies were effective for driver and passenger seats, gender, and experience (number of times).

In the future, we plan to conduct more experiments with automated vehicles other conditions, including more paths and more scenarios. In addition, we plan to expand the number of subjects and evaluate other human factors, including occupation.

\section{Data Availability}

The data used to support the findings of this study have not been made available.

\section{Conflicts of Interest}

The authors declare that they have no conflicts of interest.

\section{Acknowledgments}

This research was supported by the New Energy and Industrial Technology Development Organization.

\section{References}

[1] “US. DOT," April 2021, http://www.its.dot.gov/automated_ vehicle/index.htm.

[2] “ITS Japan," April 2021, http://www.its-jp.org/english/files/ 2015/04/SIP_Worlshop2015_leaflets_e_20150326.pdf.

[3] European Commission, "IOT large scale pilot 5 autonomous vehicles in a connected environment," April 2021, https://www. ertrac.org/uploads/documents_publications/2015\%20ART\%20 Info\%20Day/IoTPilot-autonomous\%20vehicle-nov5.pdf.
[4] M. Aeberhard, S. Rauch, M. Bahram et al., "Experience, results and lessons learned from automated driving on Germany's highways," IEEE Intelligent Transportation Systems Magazine, vol. 7, no. 1, pp. 42-57, 2015.

[5] S. Shladover, "Challenges to evaluation of $\mathrm{CO} 2$ impacts of intelligent transportation systems," in Proceedings of the 2011 IEEE Integrated and Sustainable Transportation System, Vienna, Austria, June-July 2011.

[6] S. Tsugawa, S. Jeschke, and S. E. Shladover, "A review of truck platooning projects for energy savings," IEEE Transactions on Intelligent Vehicles, vol. 1, no. 1, pp. 68-77, 2016.

[7] R. Hoeger, A. Amditis, M. Kunert et al., "Highly automated vehicles for intelligent transport: HAVEit approach," in Proceedings of the 15th ITS World Congress, New York, NY, USA, November 2008.

[8] F. O. Flemisch, K. Bengler, H. Bubb, H. Winner, and R. Bruder, "Towards cooperative guidance and control of highly automated vehicles: H-mode and conduct-by-wire," Ergonomics, vol. 57, no. 3, pp. 343-360, 2014.

[9] E. Boer and P. Spyridakos, "Control-theoretic attentionswitching driver model," in Proceedings of the International Conference on Traffic and Transport Psychology, Brisbane, Australia, August 2016.

[10] M. Omae, N. Hashimoto, T. Sugamoto, and H. Shimizu, "Measurement of driver's reaction time to failure of steering controller during automatic driving," Review of Automotive Engineering, vol. 26, pp. 213-215, 2005.

[11] G. M. Fitch, R. J. Kiefer, J. M. Hankey, and B. M. Kleiner, "Toward developing an approach for alerting drivers to the direction of a crash threat," Human Factors: The Journal of the Human Factors and Ergonomics Society, vol. 49, no. 4, pp. 710-720, 2007.

[12] V. Butakov and P. Ioannou, "Driving autopilot with personalization feature for improved safety and comfort," in Proceedings of the 2015 IEEE 18th International Conference on Intelligent Transportation Systems, Las Palmas de Gran Canaria, Spain, September 2015.

[13] Y. Du, C. Liu, and Y. Li, "Velocity control strategies to improve automated vehicle driving comfort," IEEE Intelligent Transportation Systems Magazine, vol. 10, no. 1, pp. 8-18, 2018.

[14] A. Reschka, J. R. Böhmer, F. Saust, B. Lichte, and M. Maurer, "Safe, dynamic and comfortable longitudinal control for an autonomous vehicle," in Proceedings of the IEEE Intelligent Vehicles Symposium, Alcala de Henares, Spain, June 2012.

[15] C. Sohn, J. Andert, and R. N. Nanfah Manfouo, “A driveability study on automated longitudinal vehicle control," IEEE Transactions on Intelligent Transportation Systems, vol. 21, no. 8, pp. 3273-3280, 2020.

[16] M. Park, S. Lee, and W. Han, "Development of lateral control system for autonomous vehicle based on adaptive pure pursuit algorithm," in Proceedings of the 2014 14th International Conference on Control, Automation and Systems, Gyeonggi-do, South Korea, October 2014.

[17] G. Lee, S. Kim, Y. Yim, J. Jung, S. Oh, and B. Kim, "Longitudinal and lateral control system development for a platoon of vehicles," in Proceedings of the 1999 IEEE/IEEJ/JSAI International Conference on Intelligent Transportation Systems, Tokyo, Japan, October 1999.

[18] B.-C. Chen, C.-T. Tsai, and K. Lee, "Path-following steering controller of automated lane change system with adaptive preview time," in Proceedings of the 2015 IEEE International Conference on Systems, Man, and Cybernetics, Kowloon, Hong Kong, October 2015. 
[19] M. Yamakado and M. Abe, "Proposal of the longitudinal driver model in coordination with vehicle lateral motion based upon jerk information," in Proceedings of the 2008 International Conference on Control, Automation and Systems, Seoul, South Korea, October 2008.

[20] A. Alleyne and M. DePoorter, "Lateral displacement sensor placement and forward velocity effects on stability of lateral control of vehicles," in Proceedings of the 1997 American Control Conference, Albuquerque, New Mexico, June 1997.

[21] P. Hingwe and M. Tomizuka, "Experimental evaluation of a chatter free sliding mode control for lateral control in AHS," in Proceedings of the 1997 American Control Conference, Albuquerque, New Mexico, June 1997.

[22] Z. Wang, R. Zheng, T. Kaizuka, and K. Nakano, "The effect of haptic guidance on driver steering performance during curve negotiation with limited visual feedback," in Proceedings of the 2017 IEEE Intelligent Vehicles Symposium (IV), pp. 600-605, Redondo Beach, CA, USA, June 2017.

[23] C. Sentouh, A.-T. Nguyen, M. A. Benloucif, and J.-C. Popieul, "Driver-automation cooperation oriented approach for shared control of lane keeping assist systems," IEEE Transactions on Control Systems Technology, vol. 27, no. 5, pp. 1962-1978, 2019.

[24] S. Chen, S. H. Li, B. Ma, and Z. Ran, "Ride comfort control of electrical vehicle in the process of speed regulation," in Proceedings of the 2006 6th World Congress on Intelligent Control and Automation, Dalian, China, June 2006.

[25] A. Zuska and D. Więckowski, "The impact of unbalanced wheels and vehicle speed on driving comfort," in Proceedings of the 2018 XI International Science-Technical Conference Automotive Safety, Častá, Slovakia, April 2018.

[26] I. Bae, J. H. Kim, J. Moon, and S. Kim, "Lane change maneuver based on Bezier curve providing comfort experience for autonomous vehicle users," in Proceedings of the 2019 IEEE Intelligent Transportation Systems Conference (ITSC), Auckland, New Zealand, October 2019.

[27] J. Xie, J. Gong, S. Wu, G. Xiong, and C. Lu, "A personalized curve driving model for intelligent vehicle," in Proceedings of the 2017 IEEE International Conference on Unmanned Systems (ICUS), Beijing, China, October 2017.

[28] O. Lee, A. Rasch, A. L. Schwab, and M. Dozza, "Modelling cyclists' comfort zones from obstacle avoidance manoeuvres," Accident Analysis \& Prevention, vol. 144, p. 105609, Article ID 105609, 2020.

[29] L. Oliveira, K. Proctor, C. G. Burns, and S. Birrell, "Driving style: how should an automated vehicle behave?" Information, vol. 10, no. 6, p. 219, 2019.

[30] F. L. Mrad, D. M. L. Machado, G. J. C. Horta, and A. U. Sad, "Optimization of the vibrational comfort of passenger vehicles through improvement of suspension and engine rubber mounting setups," Shock and Vibration, vol. 2018, pp. 1-9, Article ID 9861052, 2018

[31] A. Sezgin and N. Yagiz, "Analysis of passenger ride comfort," in Proceedings of the International Conference on Structural Nonlinear Dynamics and Diagnosi MATEC Web of Conferences, vol. 1, Marrakech, Morocco, April-May 2012.

[32] "ISO standard 2631," August 2021, https://www.iso.org/ standard/76369.html.

[33] Z. Htike, G. Papaioannou, E. Siampis, E. Velenis, and S. Longo, "Minimisation of motion sickness in autonomous vehicles," in Proceedings of the 2020 IEEE Intelligent Vehicles Symposium, pp. 1135-1140, Nagoya, Japan, July 2020.

[34] D. Li and J. Hu, "Mitigating motion sickness in automated vehicles with frequency-shaping approach to motion planning," IEEE Robotics and Automation Letters, vol. 6, no. 4, pp. 7714-7720, 2021.

[35] S. Sedighi, D. V. Nguyen, and K. D. Kuhnert, "A new method of clothoid-based path planning algorithm for narrow perpendicular parking spaces," in Proceedings of the Paper Presented at Proceedings of ICMRE 2019, Rome, Italy, February 2019.

[36] P. F. Lima, M. Trincavelli, J. Mårtensson, and B. Wahlberg, "Clothoid-based model predictive control for autonomous driving," in Proceedings of the Paper Presented at 2015 European Control Conference (ECC), Linz, Austria, July 2015.

[37] T. Berglund, A. Brodnik, H. Jonsson, M. Staffanson, and I. Soderkvist, "Planning smooth and obstacle-avoiding B-spline paths for autonomous mining vehicles," IEEE Transactions on Automation Science and Engineering, vol. 7, no. 1, pp. 167-172, 2010.

[38] T. De-rong and Y. Peng, "Study on the forecast method of road accidents based on extension of cubic spline curve," in Proceedings of the Paper Presented at 2009 Second International Conference on Intelligent Computation Technology and Automation, Changsha, China, October 2009.

[39] Y. Wang, P. Cai, and G. Lu, "Cooperative autonomous traffic organization method for connected automated vehicles in multi-intersection road networks," Transportation Research Part C: Emerging Technologies, vol. 111, pp. 458-476, 2020.

[40] SAE International, Automated Driving-Levels of Driving Automation are Defined in New SAE International Standard J3016, SAE International, Warrendale, PA, USA, 2021, https:// www.sae.org/misc/pdfs/automated_driving.pdf.

[41] N. Montes, A. Herraez, L. Armesto, and J. Tornero, "Real-time clothoid approximation by Rational Bezier curves," in Proceedings of the 2008 IEEE International Conference on Robotics and Automation, pp. 2246-2251, Pasadena, CA, USA, May 2008.

[42] M. Yamamoto and S. Nishiyama, "Proposal for a transition curve with smoothly changing curvature using multiple clothoid curve," Transactions of the JSME, vol. 85, no. 873, 2019, in Japanese.

[43] D. J. Walton and D. S. Meek, "A controlled clothoid spline," Computers \& Graphics, vol. 29, no. 3, pp. 353-363, 2005.

[44] V. Girbés, G. Vanegas, and L. Armesto, "Clothoid-based three-dimensional curve for attitude planning," Journal of Guidance, Control, and Dynamics, vol. 42, no. 8, pp. 18861898, 2019.

[45] N. Yusof, J. Karjanto, J. Terken, F. Delbressine, and M. Hassan, "The exploration of autonomous vehicle driving styles: preferred longitudinal, lateral, and vertical accelerations," in Proceedings of the 8th International Conference on Automotive User Interfaces and Interactive Vehicular Applications, pp. 245-252, Ann Arbor, MI, USA, October 2016.

[46] K. Ikeda, A. Endo, R. Minowa, T. Narita, and H. Kato, "Ride comfort control system considering physiological and psychological characteristics: effect of masking on vertical vibration on passengers," Actuators, vol. 7, no. 3, p. 42, 2018.

[47] J. Zhou, R. He, Y. Wang et al., "Autonomous driving trajectory optimization with dual-loop iterative anchoring path smoothing and piecewise-jerk speed optimization," IEEE Robotics and Automation Letters, vol. 6, no. 2, pp. 439-446, 2021.

[48] Y. Li, "Applications of chi-square test and contingency table Analysis in customer satisfaction and empirical analyses," in Proceedings of the 2009 International Conference on Innovation Management, pp. 105-107, Wuhan, China, December 2009. 
[49] B. Brumback and M. Srinath, "A Chi-square test for faultdetection in Kalman filters," IEEE Transactions on Automatic Control, vol. 32, no. 6, pp. 552-554, 1987.

[50] B. Ahmed and P. H. Holmes, "A voice activity detector using the chi-square test," in Proceedings of the 2004 IEEE International Conference on Acoustics, Speech, and Signal Processing, pp. I-625, Montreal, Canada, May 2004. 